\title{
Electromagnetic-based evaluation of different Halbach array topologies with gap consideration for the permanent magnet synchronous machines
}

\author{
Pedram Asef $^{1}$ - Ramon Bargallo Perpina ${ }^{1}$ - M. R. Barzegaran ${ }^{2}$ (1) \\ Tanushree Agarwal ${ }^{2}$
}

Received: 9 May 2017 / Accepted: 25 October 2017

(C) Springer-Verlag GmbH Germany 2017

\begin{abstract}
This paper investigates the influence of various Halbach arrays permanent magnets (PMs) on the electromagnetic performance of a radial flux machine with outer rotor topology. The static analysis is performed using a 2-D finite-element analysis (FEA) of six different machines with different Halbach array-based orientations. The gap consideration between PM segments is considered for the first time. The aim of the study is to find the most suitable magnetization topology determination for the PMSMs which brings maximum airgap flux density, coenergy, output torque turning, back-EMF, output electromagnetic power, and minimum corresponding harmonics, cogging torque and the likelihoods of saturation. Additionally, a comparative study of a continuous distribution that commercially is called polar anisotropic will be entirely discussed. The proposed model is verified by FEA, together with its experimental investigation for the small wind power generation application in the urban area. In addition, commercial and environmental issues of the project have been highly considered to reduce $\mathrm{CO}_{2}$ emissions as the part of green power generation mission.
\end{abstract}

M. R. Barzegaran

barzegaran@lamar.edu

Pedram Asef

pedram.asef@upc.edu

Ramon Bargallo Perpina

ramon.bargallo@upc.edu

Tanushree Agarwal

tagarwal@lamar.edu

1 Department of Electrical Engineering, Polytechnic University of Catalonia-BarcelonaTech, EEBE, Campus Diagonal-Besòs, Edifici A - Avingudad'EduardMaristany 10-14, 08019 Barcelona, Spain

2 Renewable energy Microgrid Laboratory, Lamar University (A Texas State University), Beaumont, TX, USA
Keywords Halbach array · Permanent magnet synchronous machines · Electromagnetics · Gap consideration · FEA

\section{List of symbols}

$L_{\mathrm{d}} \quad d$-axis inductance $(\mathrm{mH})$

$L_{\mathrm{q}} \quad q$-axis inductance $(\mathrm{mH})$

$M_{\mathrm{p}} \quad$ Ripple torque (\%)

$T_{\mathrm{r}} \quad$ Rated torque $(\mathrm{N} \cdot \mathrm{m})$

$T_{\mathrm{c}} \quad$ Cogging torque $(\mathrm{N} \cdot \mathrm{m})$

$W_{\mathrm{c}} \quad$ Coenergy $(\mathrm{J})$

$\psi \quad$ Flux linkage $(\mathrm{mVs})$

$P_{\mathrm{o}} \quad$ Output power (W)

$\alpha_{\mathrm{pa}} \quad$ The relative pole arc coefficient

$D_{\text {si }}, D_{\text {so }} \quad$ Inner and outer stator diameters (mm)

$L_{\mathrm{m}} \quad$ Thickness of permanent magnet $(\mathrm{mm})$

$D_{\text {ri }}, D_{\text {ro }} \quad$ Inner and outer rotor diameters $(\mathrm{mm})$

A Cross-sectional area of a counter $\left(\mathrm{mm}^{2}\right)$

$S_{\mathrm{d}} \quad$ Effective slot depth (mm)

$P_{\text {arc }} \quad$ PM $\operatorname{arc}\left({ }^{\circ} \mathrm{e}\right)$

$l_{\mathrm{i}} \quad$ The initial segment number per pole

$n_{\mathrm{m}} \quad$ Minimum speed (rpm)

$P_{\mathrm{EM}} \quad$ EM output rated power (W)

$n_{\mathrm{r}} \quad$ Rated speed (rpm)

$S_{\mathrm{w}} \quad$ Slot width (mm)

$M_{\mathrm{r}}, M_{\theta} \quad$ The radial and tangential components of the airgap magnetic flux density ( $\mathrm{T}$ )

$W_{\mathrm{p}} \quad$ The pole pitch

$W_{n \text { pa }} \quad$ The pole arc of the $n$th magnet pole

$W_{\mathrm{m}} \quad$ The pole arc of mid-magnet

$\left|B_{\text {radial }}^{h}\right| \quad$ The fundamental amplitude of the radial component of airgap flux density (T)

$B_{\text {radial }}^{h} \quad$ The $i$ th harmonic amplitude of the radial component of airgap flux density (T) 


\begin{tabular}{|c|c|}
\hline$J_{\mathrm{c}}$ & Current density $\left(\mathrm{A} / \mathrm{mm}^{2}\right)$ \\
\hline$l_{\mathrm{s}}$ & Active stack stator length \\
\hline$m$ & Stator number of phases \\
\hline$N$ & Number of turns per phase \\
\hline $2 p, p$ & Number of poles and poles pair \\
\hline$Q_{\mathrm{s}}$ & Total number of the stator slots \\
\hline$W_{\mathrm{ph}-\mathrm{s}}$ & Width of phase separator (excluding legs) (mm) \\
\hline$L_{\mathrm{a}-\mathrm{pm}}$ & Airgap between PMs (mm) \\
\hline$\delta_{\mathrm{g}}$ & Airgap length between stator and rotor $(\mathrm{mm})$ \\
\hline$\delta_{\mathrm{gs}}$ & Sides gap between each PM's segment (mm) \\
\hline$\delta_{\mathrm{gm}}$ & Middle gap between each PM's segment (mm) \\
\hline$\mu_{0}, \mu_{\mathrm{r}}$ & $\begin{array}{l}\text { The permeability of airgap, and the relative recoil } \\
\text { permeability of PM }\end{array}$ \\
\hline$A_{1}, A_{2}$ & $\begin{array}{l}\text { The distance between PMs, and the width seg- } \\
\text { ment }\end{array}$ \\
\hline$B_{\mathrm{g}}$ & Airgap magnetic flux density $(\mathrm{T})$ \\
\hline$H_{\mathrm{g}}$ & Airgap magnetic flux intensity (At/m) \\
\hline$H_{\mathrm{PM}}$ & $\begin{array}{l}\text { Magnetic flux intensity produced by magnets } \\
(\text { At } / \mathrm{m})\end{array}$ \\
\hline$M$ & $\begin{array}{l}\text { The amplitude of magnetization vector in polar } \\
\text { coordination }(\mathrm{T})\end{array}$ \\
\hline$\theta_{i}$ & The angle between the center of the $i$ th segment \\
\hline$R_{0}, R_{1}$ & Inner and outer radii of the magnet $(\mathrm{mm})$ \\
\hline$T_{\mathrm{ph}}$ & The number of series turns per phase \\
\hline$k_{\mathrm{w} 1}$ & The fundamental harmonic winding factor \\
\hline$\Phi_{\mathrm{m} 1}$ & The fundamental magnet flux per pole (Wb) \\
\hline$\omega_{\mathrm{m}}$ & Mechanical angular velocity (mech. $\mathrm{Rad} / \mathrm{s}$ ) \\
\hline 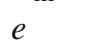 & Instantaneous EMF (V) \\
\hline
\end{tabular}

\section{Introduction}

Electromagnetic (EM) solutions possess many technical advantages contrary to conventional topologies in terms of performance of electrical machines using the HABO that results in the improvement and increase in sinusoidal electromotive forces (EMFs) waveforms which originate from an increased sinusoidal airgap flux density distribution, and reduced machine's mass because of its self-shielding magnetization that allows the machine to operate without any back iron [1-6]. The main objective is to determine the best possible magnetization orientation with the gap consideration between magnetic segments particularly in the HABOs for the first time. Therefore, the influence of different segment numbers per pole for fundamental amplitude, waveform distortion factor and all other fundamental EM aspects is studied by given objective design function (10) in Sect. 3.

In 1973, the influence of installing magnetic blocks in a certain order was discovered by Mallinson [1] to achieve higher airgap flux density for the very first time. Afterward,
Halbach [2] invented the HABO to focus particle accelerator beams in 1980s. Since then, many researchers studied analytical and numerical modeling of different HABOs in electrical machines.

The outer rotor surface mounted PM synchronous machines (PMSMs), in which a double-layer second-grade fractional winding is employed due to a number of advantages such as short end-winding. The outer rotor machines can reach a high torque at a low speed for the direct-drive applications with low-speed operation, such as small power generation [3].

Shen and Zhu [4] discussed a general analytical model which is capable of predicting the electromagnetic performance of slotted/slotless brushless PMSMs with both even- and odd-segment Halbach array, having different magnet remanence, magnetization angle and arc for each single-magnet segment. The emphasis has been on the magnetization which is the key in analytical modeling of PMSMs having a segmented Halbach array and its investigation.

Reference [5] investigated the differences between a segmented HABO PMSMs and a single-ring HABO PMSMs at airgap magnetic field distribution and back-EMF waveforms, where the gap between segments is ignored by FE model. Research has been carried out on the effects of the segment number per pole on airgap magnetic field distribution and back-EMF waveforms.

In Refs. [6], and [7], the established analytical models aimed on the Halbach array, in which it has two segments per pole and the magnetized directions of two segments are radial and tangential, respectively. Additionally, influences of the width ratio of two segments per pole on torque pulsation and maximum torque has been analyzed.

Xia et al. [8] also studied analytical modeling of HABO in PMSMs with consideration of gap between segments. Moreover, the effects of the parameters of the segmented Halbach array, enclosing gap between segments, segment number per pole and pole pair number on the fundamental amplitude, and waveform distortion factor of the radial component of airgap flux density are analyzed using the analytical model. Reference [9] presents a comparative study of torque analysis for PMSMs couplings with parallel and four-segmented Halbach array-magnetized magnets, by analytical field calculations. On the basis of the magnetic vector potential, they derived analytical solutions for the magnetic field produced by the interaction between the inner and outer PMs for the parallel array- and Halbach array-magnetized cases. Then, the magnetic torque calculation using the derived solutions and Maxwell stress tensor have been reported. This study inspired us to consider gap consideration for the most wellknown HABO's topologies on PMSM.

Del-Valle et al. [13] investigated the levitation and guidance forces calculation and stability arising from both conventional arrangements and recently proposed Halbachlike arrangements. Afterward, a comparison was carefully 
made under similar conditions, which conclude that not always complicated arrangements based on Halbach arrays bring significant improvements with respect to some simpler arrangements that also provide large force.

This paper aims to gauge EM performance for six distinctive patterns of magnetization, five HABO FE models and one conventional parallel-based FE model. The paper also analyzes the way the modified 2-segmented halfback array develops its performance in comparison with the classic 2segmented halfback array model for the outer rotor PMSM with a double-layer and 2nd-grade fractional slot winding $[10,11]$. All the significant EM perspectives are performed with the gap consideration among the FE models using a design function with a number of constraints. At last, the best magnetization topology is proposed for experimental investigation. Additionally, the proposed prototype is under operation for a standalone wind energy conversion system [12].

The rest of paper is spanned into four sections. In Sect. 3, EM analytical design definition, is discussed analytical standpoint of various magnetization topologies. In addition, a design function with a number of constraint is introduced to find the best possible size of the gaps between magnets. Afterward, EM-based results and discussion section investigated numerically using 2-D FEA how the capability of EM analysis weighted into a considerable comparative study in terms of radial and tangential components of airgap flux density, flux linkage waveforms, back-EMF, corresponding accurate calculation of the harmonics, cogging torque, output torque turning, iron loss, total harmonic distortion (THD), and the output power. Moreover, the effect of segment number per pole on the fundamental amplitude and distortion waveform parameter of the radial component, as well as the likelihood of saturation are considered. In Sect. 5, the manufactured prototype is discussed. The conclusion as the last section provided the outcomes of the research.

\section{EM analytical design definition}

In EM design, it is always crucial to deal with various levels of performance and its complexity. However, based on the demand, the design topology and quality may change.

The key in the analytical modeling of PMSMs with segmented Halbach array is the magnetization with consideration of the gap, in order to deal with interaction force between segments. The field vector in airgap and magnetic blocks (PMs) [4] can be calculated using

$$
\begin{aligned}
B_{\mathrm{g}} & =\mu_{0} \cdot H_{\mathrm{g}} \\
B_{\mathrm{PM}} & =\mu_{0} \cdot \mu_{r} \cdot H_{\mathrm{PM}}+\mu_{0} \cdot M
\end{aligned}
$$

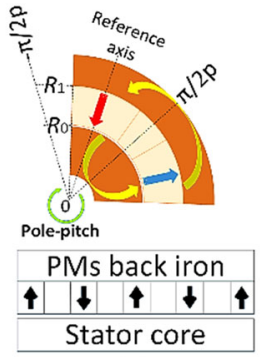

(a)

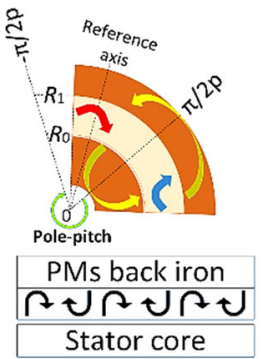

(b)

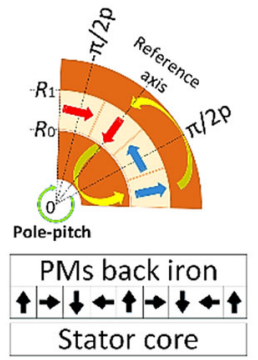

(c)

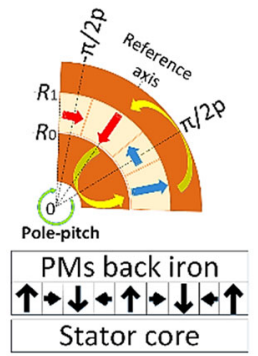

(d)

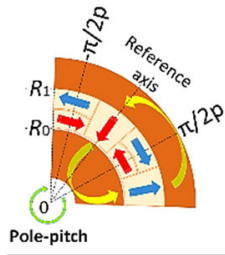

PMs back iron

Stator core

(e)

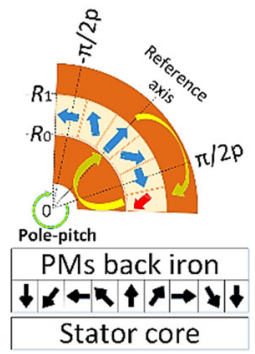

(f)

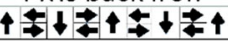

Fig. 1 A comparative schematic of different EM orientation topologies with equivalent pole pitch, a conventional parallel topology with considering airgap between magnets, b polar anisotropic topology, c 2-segmented HABO with gap consideration between magnets, d 2-segmented unbalanced HABO with gap consideration between magnets, e modified 2-segmented HABO with gap consideration between magnets and $\mathbf{f}$ 4-segmented HABO with gap consideration between magnet

where the amplitude of magnetization vector $M$ in polar coordination for parallel topology can be defined in (3)

$$
\begin{aligned}
M_{\text {parallel }}= & M_{r} \cdot r+M_{\theta} \cdot \theta=\sum_{n=1,2,3, \ldots}^{\infty} M_{r n} \cdot \cos (n p \theta) \cdot r \\
& \mp \sum_{n=1,2,3, \ldots}^{\infty} M_{\theta n} \cdot \sin (n p \theta) \cdot \theta
\end{aligned}
$$

where ' + ' has to present while the PMSM operates with outer rotor topology, which is the case study of the paper. Also, '-' for interior rotor machines [4]. In Fig. 1, a proper view of the magnetization topologies, which are used in this study for PMSM based on magnetic orientations of the PMs, segmentations, and gap considerations, can be seen. Figure 1a shows a conventional parallel EM orientation topology (CPT) using 20 pole pair $\mathrm{NdFeBr}$ magnets without any segmentation, in which gap consideration is neglected and therefore a higher demand of magnet volume needed in comparison with HABO-based models. Figure $1 \mathrm{~b}$ illustrates continuous HABO which is known as PA commercially, where no segmentation is needed to provide HABO. However, there is significant negligence that is addressed in EM-based study results and discussion section. Figure 1c presents a 2-segmented HABO with gap consideration between seg- 


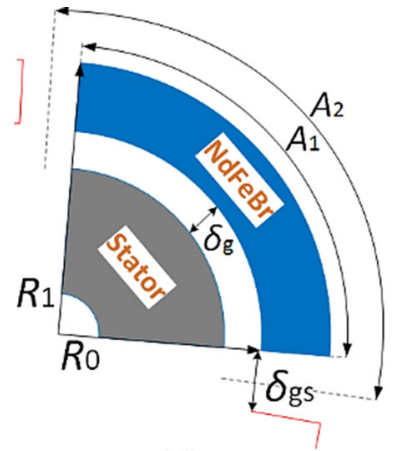

(a) (b)

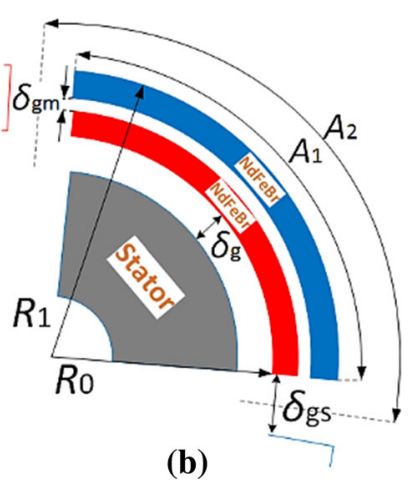

Fig. 2 Permanent magnet's geometrical design perspective with gap consideration

mented magnets. From manufacturing process standpoint, the radial gaps between magnet segments can be glued. Moreover, the interaction force between segments can be considered. Afterward, an unbalanced 2-segmented HABO with unequal area of magnet is studied which can be seen in Fig. 1d. At last, a modified 2-segmented HABO with orientation with radial and axial segmentations for gap consideration is shown in Fig. 1e. To provide a comprehensive study over the most well-know HABOs, a 4-segmented HABO with radial gap between segments is considered that is illustrated in Fig. 1f. Figure 2 represents the significant geometrical design parameters which are taken into account for the gap consideration.

In $\mathrm{HABO}$, each magnetic block has a specific magnetization orientation, where the orientation of each block can be given as

$\theta_{m, i}=\theta_{i}(1+p)$

where $\theta_{i}$ represents the angle between the center of the $i$ th segment in which $\theta=0$ (the conditional angle of orientation). Thus, $\theta_{i}$ can be redefined as follows

$\theta_{i=1,2,3, \ldots, 2 p l}=\frac{(i-1) \pi}{p \cdot l}$

The HABO consists of three segments, side magnet, midmagnet and end side magnet. Each segment is magnetized in parallel orientation, however with different angle of orientation. Therefore, the ratio of the $n$th PM pole arc to the pole pitch can be expressed as

$R_{\mathrm{pa} / \mathrm{pp}}=W_{m} / W_{p}$

The ratio of $n$th PM pole arc to the pole pitch is given

$R_{n_{\mathrm{pa} / \mathrm{pp}}}=W_{n_{\mathrm{pa}}} / W_{p}$
The gap consideration as shown in Fig. 1 can be defined by the distance between magnets presented as $A_{1}$, and the width of the segment presented as $A_{2}$. Thus, the ratio of $A_{1}$ to $A_{2}$ is defined as the relative pole arc coefficient using (8)

$\alpha_{\mathrm{pa}}=A_{2} / A_{1}$

Accordingly, the gap between PM segments in this paper is calculated by the relative pole arc coefficient and the radius at the center of the PM [7], given by

$\Delta A=A_{2}-A_{1}=\left(1-\alpha_{\mathrm{pa}}\right) \cdot \frac{\pi\left(R_{0}+R_{1}\right)}{2 p \cdot l}$

Normally, in many papers the consideration of gap between segments was neglected in order to reduce design complexity by setting to one. Consequently, the gap between magnetic blocks will be zero.

To determine the best gap size between magnet segments, the following objective design functions can be drawn into an optimization process

$$
\text { s.t. }\left\{\begin{array}{l}
f_{1}(x)=\left(1-\alpha_{\mathrm{pa}, i}\right) \cdot \frac{\pi\left(R_{0}(i)+R_{1}(i)\right)}{2 p \cdot l} \\
g_{1}(x)=450-P_{\mathrm{out}} \leq 0 \\
g_{2}(x)=J_{c}-4 \leq 0 \\
g_{3}(x)=T_{r}(i)-T_{r n} \leq 0 \\
g_{4}(x)=T_{c}(i)-T_{c n} \leq 0 \\
g_{5}(x)=B_{i}-2.1 \leq 0 \\
g_{6}(x)=0.92-\eta \leq 0 \\
g_{7}(x)=s f-0.7 \leq 0
\end{array}\right.
$$

Through this section, the conceptual idea of the paper with introducing the magnetization topologies and its considerations was presented. Next, the EM comparative results are discussed in following section.

\section{EM-based study results and discussion}

According to the preceding sections, the magnetic flux density distribution affects predominantly on maximizing the airgap flux density of models. This leads to higher output torque and power. In this section, analytical calculations will be validated through FEA and, in addition, both calculation methodologies are implemented with the considerable accuracy. The airgap flux density [14] can be calculated as follows

$$
\begin{aligned}
B_{r}= & \sum_{n} \frac{q\left(M_{n} \cdot H_{n}+N_{n} \cdot K_{n}\right)}{\mu_{r}\left(q^{2}-1\right)} \\
& \cdot\left[r^{q-1}+a^{2 q} r^{-q-1}\right] c^{-q+1} H_{n} \cos (q \theta)
\end{aligned}
$$

where $q=n p ; M_{n}$ and $N_{n}$ are the $n$th harmonic component of the radial and tangential components of magnetization; $H_{n}$ 


\begin{tabular}{|l|l|}
\hline & $1.930 \mathrm{e}+000:>2.000 \mathrm{e}+000$ \\
$1.860 \mathrm{e}+000: 1.930 \mathrm{e}+000$ \\
$1.790 \mathrm{e}+000: 1.860 \mathrm{e}+000$ \\
$1.720 \mathrm{e}+000: 1.790 \mathrm{e}+000$ \\
$1.650 \mathrm{e}+000: 1.720 \mathrm{e}+000$ \\
$1.580 \mathrm{e}+000: 1.650 \mathrm{e}+000$ \\
$1.510 \mathrm{e}+000: 1.580 \mathrm{e}+000$ \\
$1.440 \mathrm{e}+000: 1.510 \mathrm{e}+000$ \\
$1.370 \mathrm{e}+000: 1.440 \mathrm{e}+000$ \\
$1.300 \mathrm{e}+000: 1.370 \mathrm{e}+000$ \\
$1.230 \mathrm{e}+000: 1.300 \mathrm{e}+000$ \\
$1.160 \mathrm{e}+000: 1.230 \mathrm{e}+000$ \\
$1.090 \mathrm{e}+000: 1.160 \mathrm{e}+000$ \\
$1.020 \mathrm{e}+000: 1.090 \mathrm{e}+000$ \\
$9.500 \mathrm{e}-001: 1.020 \mathrm{e}+000$ \\
$8.800 \mathrm{e}-001: 9.500 \mathrm{e}-001$ \\
$8.100 \mathrm{e}-001: 8.800 \mathrm{e}-001$ \\
$7.400 \mathrm{e}-001: 8.100 \mathrm{e}-001$ \\
$6.700 \mathrm{e}-001: 7.400 \mathrm{e}-001$ \\
$<6.000 \mathrm{e}-001: 6.700 \mathrm{e}-001$
\end{tabular}

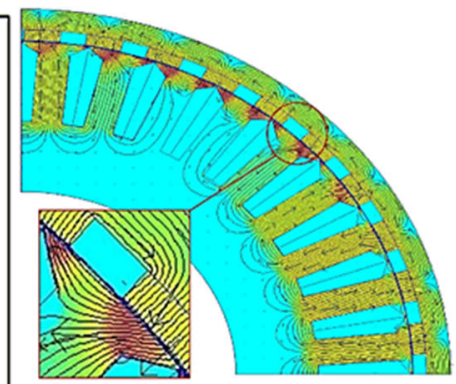

(a)

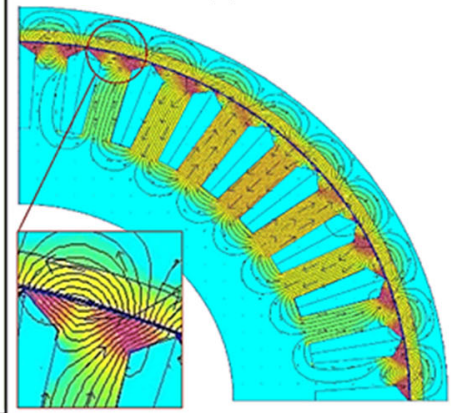

(b)

Fig. 3 Magnetic flux density distribution using FEA for different magnetized PM topologies with equivalent pole pitch, a conventional parallel topology considering the airgap between magnets, $\mathbf{b}$ polar anisotropic topology, $\mathbf{c} 2$-segmented HABO with the gap consideration

and $k_{n}$ are functions of $q, \mu_{r}$ and the different radii given in Rasmussen's paper [14]. The back-EMF as an open-circuit concept is defined with [17]

$e=\frac{\partial \psi}{\partial t}=\omega \cdot \frac{\partial \psi}{\partial \theta}$

Accordingly, the fundamental EMF/phase is calculated using (13)

$E_{q 1}=\left(\frac{p \cdot k_{w 1} \cdot T_{p h} \cdot \Phi_{m 1}}{\sqrt{2}}\right) \cdot \omega_{m}$

The flux linkage is $[16,17]$

$\psi=\int \mathrm{edt}$

Based on Fig. 3, it is obvious that by implementing various magnetization topologies, the pole pair of the machine yet remained unchanged. The veracity of magnetic fields with a maximum value of $1.8(\mathrm{~T})$ somewhere around magnetic blocks can be seen in purple color. In Fig. 4a, it can be seen that $\mathrm{PA}$ is provided a great sinusoidal radial component which is utilized to model $\mathrm{HABO}$ without considering segmentation and gaps with a significant error in comparison with the other real cases of HABOs. Furthermore, the error also can be

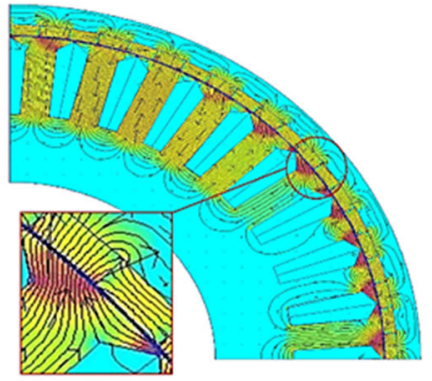

(c)

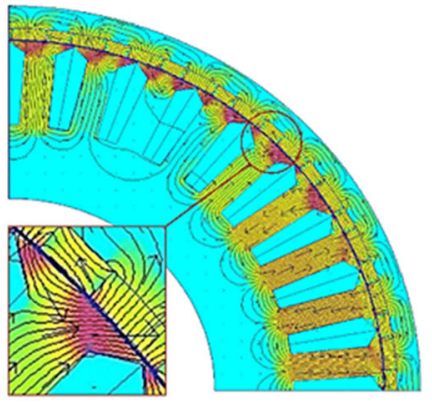

(d)

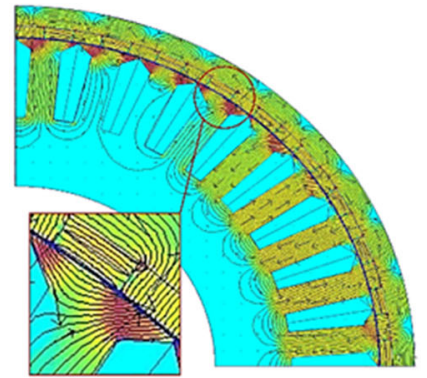

(e)

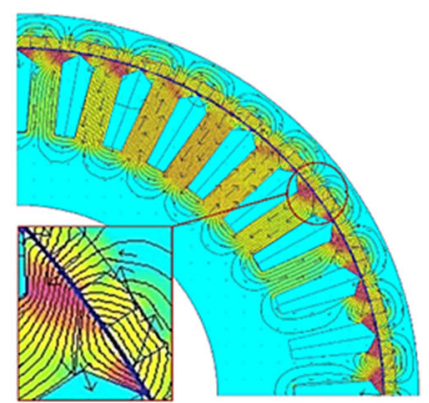

(f) between magnets, $\mathbf{d} 2$-segmented unbalanced HABO topology with the gap consideration between magnets, e modified 2-segmented HABO with the gap consideration between magnets and $\mathbf{f}$ 4-segmented HABO with the gap consideration between magnets

seen in the tangential component of the magnetic airgap flux density.

According to the results, it should be mentioned that the modified 2-segmented and 4-segmented models presented a better probity among all HABOs. Unbalanced 2-segmented and 4-segmented models recorded bigger radial and tangential components of the airgap flux density. The waveform distortion factor of the radial component of airgap flux density [7] is achieved by (15)

$K_{\mathrm{dB}}=\sqrt{\sum_{i \neq 1}\left(\frac{\left|B_{\text {radial }}^{1}\right|}{B_{\text {radial }}^{h}}\right)^{2}}$

The gap between segments should not be changed to study the effect of the segment number per pole on the radial component of airgap flux. In Eq. 9, if only the gap between segments is unchangeable under the condition for the radius of magnet, pole pair number will be constant. Therefore, the relative pole arc coefficient [7] will change with the segment number per pole through below expression

$\alpha_{\mathrm{pa}}=1-\frac{l}{l_{i}}\left(1-\alpha_{\mathrm{pa}}^{i}\right)$ 


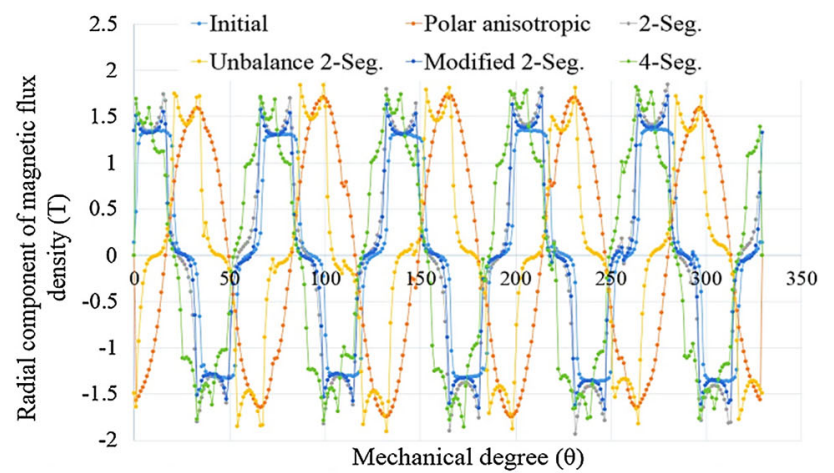

(a)

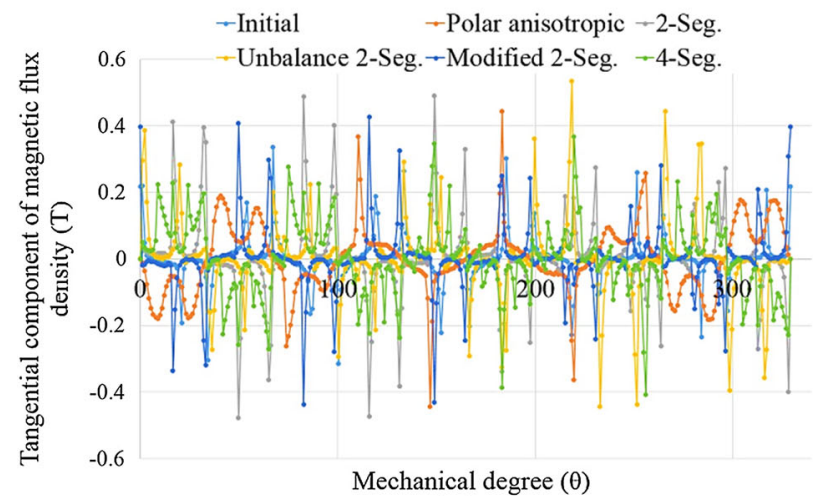

(b)

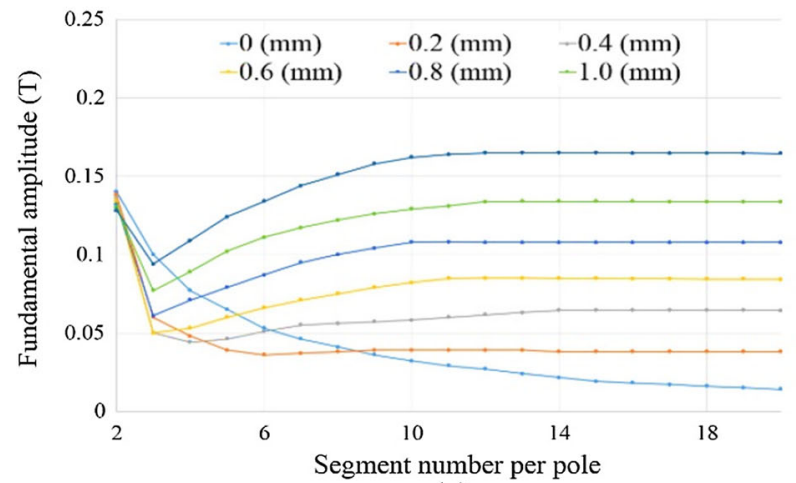

(c)

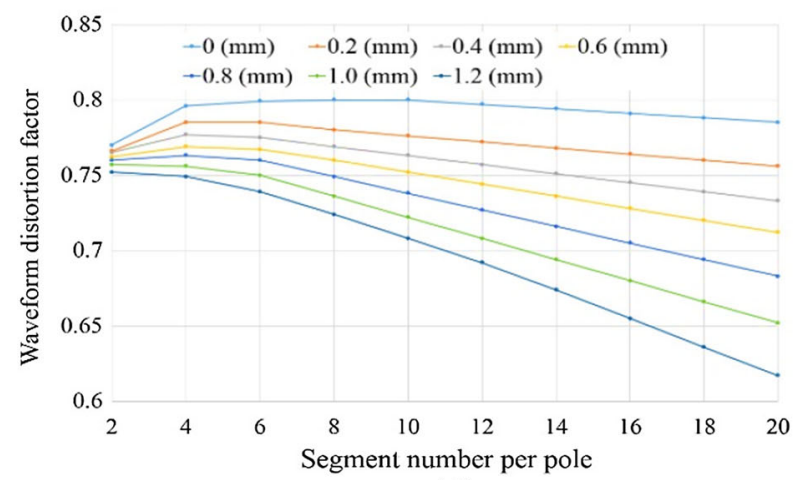

(d)

Fig. 4 FE comparison of the magnetic airgap flux density capability with gap consideration, a radial component of the magnetic flux density, b tangential component of the magnetic flux density, c effect of segmentation on waveform distortion factor, and $\mathbf{d}$ effect of segmentation on fundamental amplitude

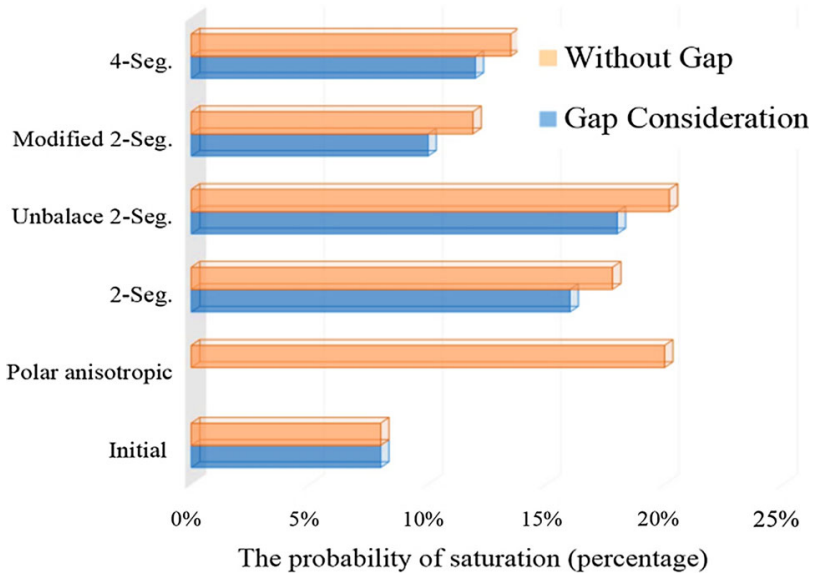

Fig. 5 Probability of magnetic flux density saturation

Figure $4 \mathrm{c}$ demonstrates that the fundamental amplitude of the radial component of airgap flux density is increased slowly and its stability is resulted from the increase in the segment number per pole, while the gap between segments is chosen to zero. The fundamental amplitude of the radial component of airgap flux density reaches the maximum value at the beginning and subsequently a gradual reduction via the increase in the segment number per pole when the gap between segments sets involving a value other than zero and the consideration of the gap between segments is accounted. In addition, when a larger gap between segments is involved, the lesser segment number per pole causes the maximum fundamental amplitude, and accordingly a larger change rate of the fundamental amplitude will be carried out. Figure $4 d$ illustrates that having the gap between segments sets to zero, the waveform distortion factor of the radial component of airgap flux density is decreased slowly and it being stable is ended by the increase in the segment number per pole. In contrast, while the gap between segments involves all values except zero, the radial component of airgap flux density initially increases gradually but later on changes based on the increment of the segment number per pole. Nonetheless, the larger the gap between segments will be, the less the segment number per pole is required.

The waveform distortion factor gains a smaller value; also a larger the change rate of the waveform distortion factor causes the waveform distortion factor to be the minimum. The likelihood of the saturation (from Fig. 3 data) based on percentage is represented in Fig. 5. The CPT model has the minimum probability of magnetic saturation in comparison with the HABO models, due to use of lesser magnets. Among the HABO models, the modified 2-segmented model has the least likelihood of saturation. However, the conventional parallel orientation with lesser magnetic blocks has even less probability. Therefore, the modified 2-segmented model is proposed, while the PA magnetization even by neglecting 
segmentation and gap between blocks is observed as the highest likelihood of the EM saturation. Figure 6a shows the flux linkage waveforms from different models, in which the flux passes by the surface $S$ to link the flux linkage of the contour integral using Stokes theorem, where the expression $[15,16]$ can be given

$\psi=\int_{S}(\nabla \times A) \mathrm{d} S=\oint A \cdot d l$

From Fig. 6a, in addition, it can be seen that the PA and 4segmented models produce and pass more flux in comparison with other models. However, all Halbach array-based models have the largest amplitude of flux linkage, which is due to involving larger coenergy because of having more magnetic blocks $(\mathrm{NdFeBr})$. The generated coenergy of different models is analytically and numerically verified in Table 1 , based on the following $[14,17]$ expression

$W_{c}=\int_{0}^{i} \psi d i$

While the self-inductance remains changeless, $\psi$ is completely produced by only the PMs. Hence, the additional $\psi$ of the winding is out of consideration. Involving Fourier series on the waveforms gives the accurate harmonics calculations on the flux linkage by Fig. 6b. The fundamental has the largest normalized value (approximately 100\%). As shown in Fig. $6 \mathrm{~b}$, further harmonic orders such as 3rd and 5th are magnified for more clear illustration, again, it can be seen that the PA model provided a better picture by having the smallest major harmonics, although out of reality that tends to the error. The 4-segmented model presented a good real-case performance with having smallest 3rd and 5th. However, modified 2 -segmented model brought almost less amplitude on the 7th order.

Figure $6 \mathrm{c}$, after the largest sinusoidal back-EMF of the PA and 4-segmented models, presents the most sinusoidal backEMF waveforms, respectively. Thus far, the 4-segmented and modified 2-segmented models brought the minimum 3 rd and 5th harmonic orders, by 7th harmonic order the CPT, the modified 2-segmented and the 2-segmented with almost the same value (about $0.1 \%$ ), the PA with $0.37 \%$, and also the 4 -segmented with $0.85 \%$. Moreover, the unbalance 2-segmented model carries the highest 7 th harmonic by $2.0 \%$. Thereupon, the minimum back-EMF harmonics are obtained by the proposed 4-segmented and modified 2segmented models. Furthermore, the minimum amplitude of harmonic orders can be seen in the PA model with a thinkable difference with other HABO models. Figure 7 a shows the cogging torque waveforms which originate from the variation of the magnetic reluctance of the stator teeth as seen through

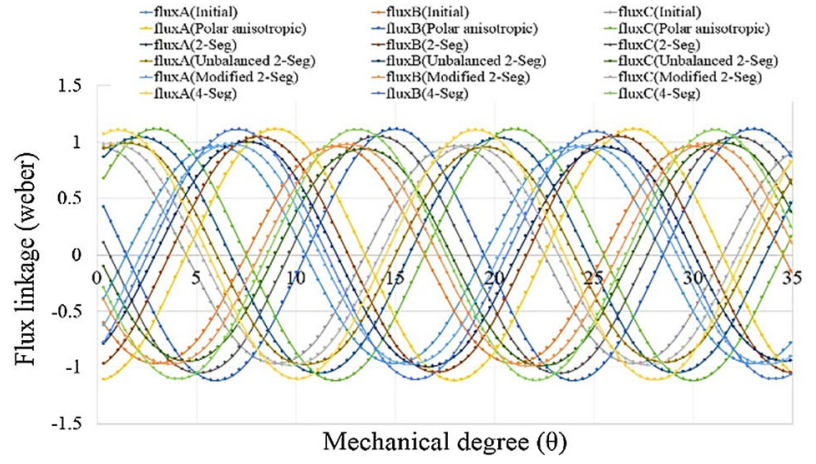

(a)

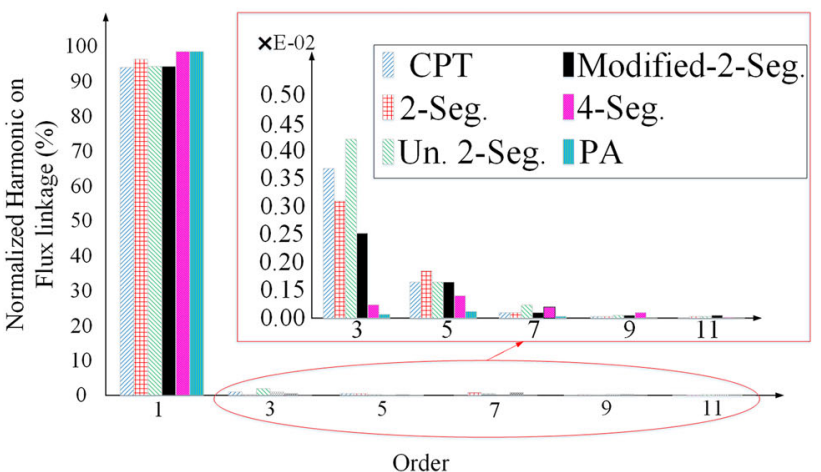

(b)

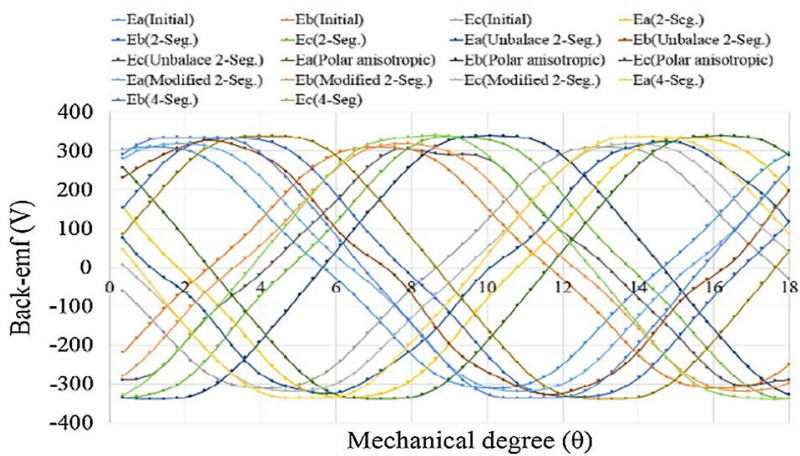

(c)

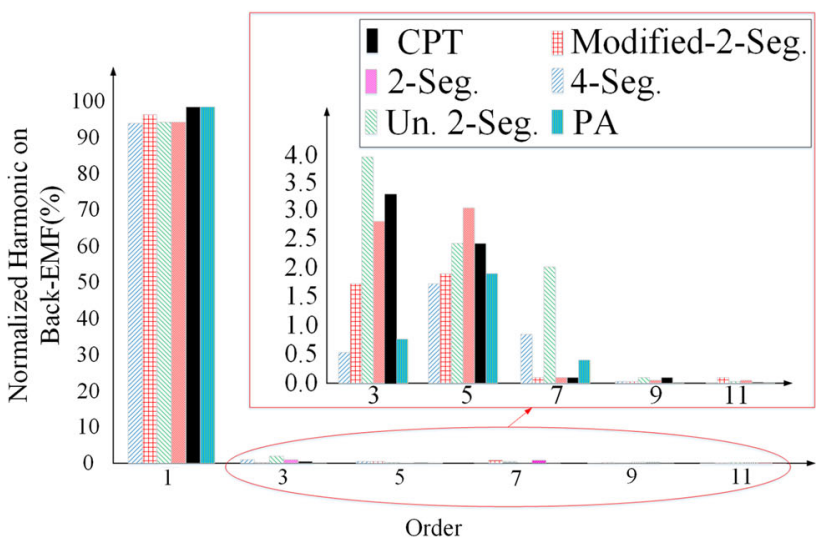

(d)

Fig. 6 FE comparison of all discussed models with gap consideration in terms of, a flux linkage waveforms, $\mathbf{b}$ harmonics on the flux linkage waveforms, $\mathbf{c}$ back-EMF waveform and $\mathbf{d}$ harmonics on the back-EMF waveforms 
Table 1 Coenergy calculation (joules)

\begin{tabular}{lcccccc}
\hline Models/methods & CPT & PA & 2-Seg. & Un. 2-seg. & Modified 2-seg. & 4-Seg. \\
\hline Analytical & 91.1 & 144.7 & 145.9 & 151 & 159.4 & 143.4 \\
FEA & 90.33 & 143.66 & 145.2 & 150.4 & 158.8 & 142.8 \\
\hline
\end{tabular}

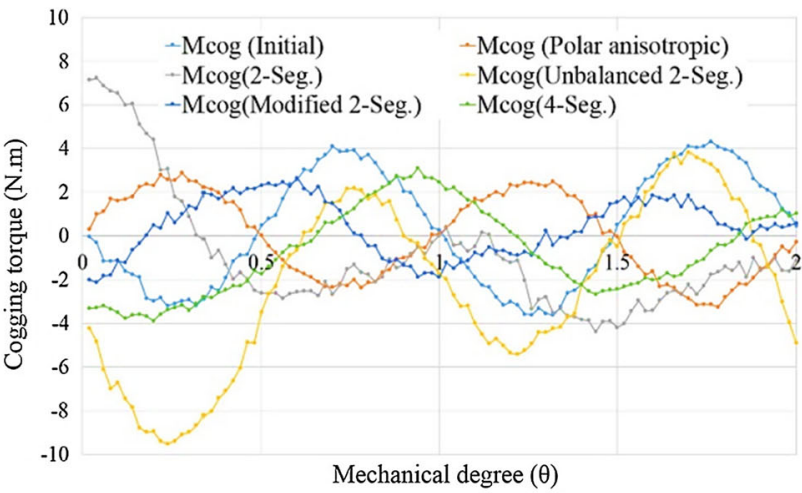

(a)

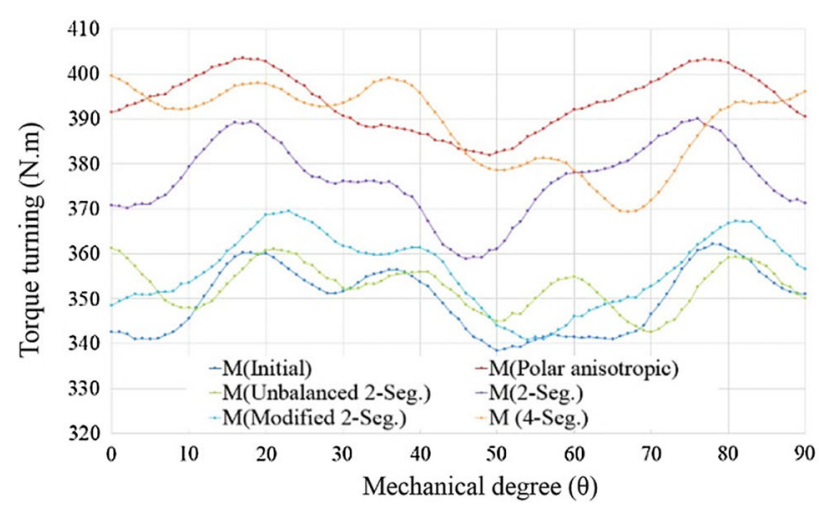

(b)

Fig. 7 FE comparison of torque calculation with gap consideration in a cogging torque waveforms and $\mathbf{b}$ torque turning waveforms

the PMs; while the rotor rotates between all models with different magnetization topologies, the modified 2-segmented model with less than $2(\mathrm{~N} \cdot \mathrm{m})$ is greatly simulated. However, the 4-segmented and PA topologies are placed as the maximum value of torque turning. Table 2 shows the comparison of the ripple torque and average torque which is calculated analytically and numerically with consideration of gap between segments $M_{p}$ (gap) and without consideration of the gap between segments $M_{p}$ (no gap). Among the results, the 4-segmented model with $390.8(\mathrm{~N} \cdot \mathrm{m})$ and $7.15 \%$ ripple torque is the most practical one. However, the unbalance 2segmented topology with the minimum ripple torque $\left(M_{p}\right)$ with $6.46 \%$ can be named as the most polished waveform. By the differences in the torque ripples, the influence of gap consideration is also obviously shown approximately $1 \%$ larger than non-gap consideration. The PA topology remains exceptional as long as this type of topology meets only theoretical issues and without any practical justification. Thus, the maximum practical average torque belongs to the 4-segmented model. The maximum EM output power with the consideration of gap for all magnetization topologies is presented in Table 3, in which the largest calculated power with the value of $601.4(\mathrm{~W})$ belongs to the theoretical PA model. The proposed topology relied on the 4-segmented design which takes the largest practical value with the consideration of the gap.

Table 4 presents the iron loss prediction comparison, where the most considerable changes belonged to the eddy current loss due to the various types of magnetization and used active magnet material. As a result, the 4-segmented model brought a minimum total iron loss of $182(\mathrm{~W})$, whereas the unbalance 2-segmented model draws on the maximum total iron loss by $241.4(\mathrm{~W})$. The calculation methodology is validated in [18-22].

Table 5 illustrates the THD over each single phase of back-EMF waveform, where it can be mentioned that mostly the highest percentage of THD is produced in the phase C in all the models. Moreover, the lowest THD is reported in the proposed 4-segmented model. Additionally, all the HABO-based models have lower THD except unbalanced 2-segmented model.

Table 2 Torque ripple calculation $(\mathrm{N} \cdot \mathrm{m})$

\begin{tabular}{llllll}
\hline Topology & Ave. torque & Analytical $M_{\mathrm{p}}($ gap) $(\%)$ & FEA $M_{\mathrm{p}}($ gap) $(\%)$ & Analytical $M_{\mathrm{p}}($ no gap) $(\%)$ & FEA $M_{\mathrm{p}}($ no gap) $(\%)$ \\
\hline CPT & 349.1 & 8.29 & 8.35 & 6.55 & 7.18 \\
PA & 394 & None & None & 5.11 & 5.87 \\
2-Seg & 376.8 & 7.22 & 8.99 & 6.31 & 7.22 \\
Unbalance 2-seg. & 353.4 & 4.88 & 6.46 & 3.62 & 5.87 \\
Modified 2-seg. & 358.7 & 7.12 & 7.89 & 5.97 & 6.94 \\
4-Seg. & 390.8 & 6.71 & 7.15 & 5.66 & 6.32 \\
\hline
\end{tabular}


Table 3 Rated EM output power $(\mathrm{W})$

\begin{tabular}{lllllll}
\hline Models/methods & CPT & PA & 2-Seg. & Un. 2-seg. & Modified 2-seg. & 4-Seg. \\
\hline Analytical & 495.9 & 600.4 & 574.6 & 539.5 & 542 & 598.7 \\
FEA & 496.2 & 601.4 & 575.1 & 541.8 & 543.3 & 600 \\
\hline
\end{tabular}

Table 4 Iron loss prediction comparison

\begin{tabular}{llll}
\hline Model & $P_{\text {hy }}(\mathrm{W})$ & $P_{\text {eddy }}(\mathrm{W})$ & $P_{\text {exe }}(\mathrm{W})$ \\
\hline CPT & 73.5 & 124.3 & 19.4 \\
PA & 60.4 & 115 & 18.3 \\
2-Seg. & 66 & 129.2 & 17.8 \\
Un. 2-seg. & 75.6 & 143.8 & 22 \\
Modified 2-seg. & 65.8 & 113.4 & 14 \\
4-Seg. & 61 & 105.3 & 15.7 \\
\hline
\end{tabular}

Table 5 THD on back-EMF per phase (\%)

Back-EMF/ CPT PA 2-Seg. Un. 2-seg. Modified 2-seg. 4-seg. models

\begin{tabular}{lllllll}
\hline A & 8.2 & 3.5 & 7.8 & 8.8 & 3.4 & 2.9 \\
B & 8.5 & 3.7 & 8.0 & 8.9 & 3.5 & 3.0 \\
C & 8.8 & 3.8 & 8.1 & 9.2 & 3.6 & 3.2 \\
\hline
\end{tabular}

\section{Experimental verification}

This section verifies the proposed HABO's FE model, in which the 4-segmented HABO model gives the best possible performance (refer to Sect. 3). Figure 8a shows the manufactured stator core with fractional slot winding, and the rotor core with proposed 4-segmented HABO is shown in Fig. 8b. Figure 9 illustrates the FEA output torque calculation (in red) with peak $150(\mathrm{Nm})$, where a modest difference of less than $2.1 \%$ justifies the error between experimental measurements and FEA results. Also, the FEA output power calculation (blue) shows $0.56(\mathrm{~kW})$ is fabulously reached with a high accuracy design at steady-state operation of the machine with minimum possible error (approximately 1.8\%). In other words, the likable experimental investigation has validated the analytical and FEA calculations, in which both torque and power as significant performance parameters have been verified.

The analytical perspective with the presentation of the gap consideration and magnetization orientations is studied. The major outcomes originated from the static FE simulation of magnetic flux density distribution over the introduced models are experimentally verified. From the EM standpoint, the waveform distortion factor and fundamental amplitude of the radial component as function of segment number per pole are discussed. For clarifying the effect of segmentation of the gap, fundamental primary EM parameters such as the

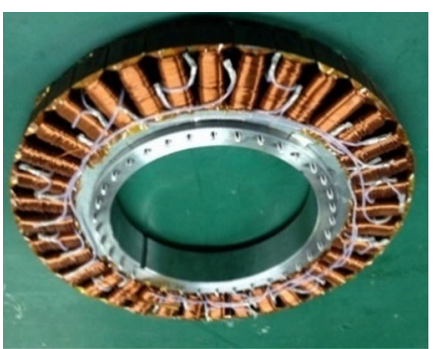

(a)

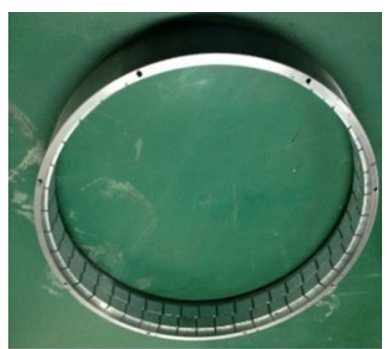

(b)
Fig. 8 Proposed 4-segmented HABO model a stator core and $\mathbf{b}$ rotor core included the PMs

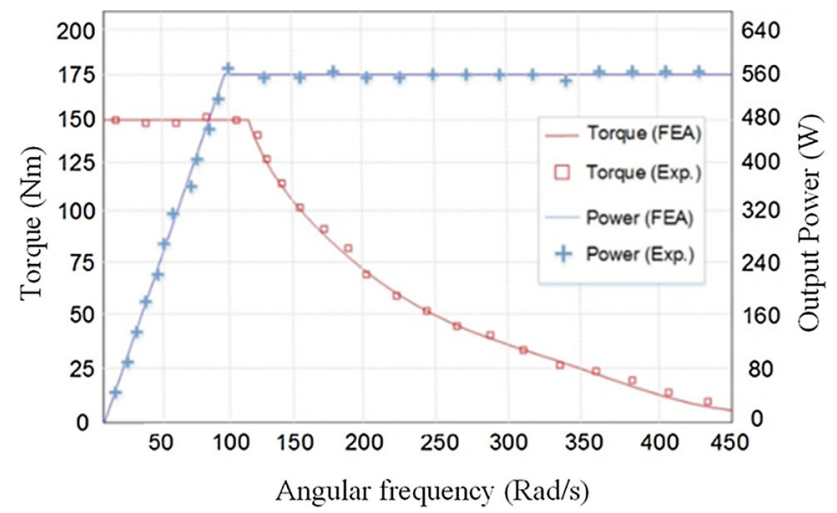

Fig. 9 FEA and experimental verifications on the mechanical torque and output electromagnetic power

radial and tangential component of airgap flux density, the flux linkage waveform, back-EMF, and their corresponding harmonics, coenergy calculation of the PMs, cogging torque, output torque turning with related torque ripple calculation, and the output EM power are comprehensively compared and discussed in the article.

\section{Conclusion}

In this paper, the electromagnetic influence of different HABOs with the consideration of the gap between PM segments is investigated for an outer rotor permanent magnet synchronous machine. Moreover, the analytical perspective with the presentation of the gap consideration and magnetization orientations is studied. According to the findings, the likelihood of EM saturation in the modified 2-segmented model has the least likelihood of saturation. From the EM standpoint, the waveform distortion factor and fundamental 
amplitude of the radial component as function of segment number per pole are discussed. Correspondingly, the use of PA topology can be useful only for very rapid prediction (pre-design) as this type of magnetization modeling neglects the gap consideration and segmentation. Thus, a significant error may exist. The major purpose of employing HABOs is to increase the airgap flux density. Following EM benefits, the achievements of the 4-segmented model are perfectly obtained, in which the results are compared and also FEA along with experimental verification resulted in an error less than $0.3 \%$.

\section{Appendix}

Machine design key parameters can be presented by the following Table 6.

Table 6 Design of the machine geometry

\begin{tabular}{lll}
\hline Variable & Value & Unit \\
\hline$D_{\text {ro }} / D_{\text {ri }}$ & $460 / 430$ & $\mathrm{~mm}$ \\
$D_{\text {so }} / D_{\text {si }}$ & $419 / 228$ & $\mathrm{~mm}$ \\
$l_{\mathrm{s}}$ & 100 & $\mathrm{~mm}$ \\
$A_{\text {slot }}$ & 802.7 & $\mathrm{~mm}^{2}$ \\
$L_{\mathrm{m}}$ & 8 & $\mathrm{~mm}$ \\
$\delta_{\mathrm{g}}$ & 0.6 & $\mathrm{~mm}$ \\
$S_{\mathrm{w}}$ & 15 & $\mathrm{~mm}$ \\
$P_{\mathrm{arc}}$ & 100 & $\circ$ \\
$S_{\mathrm{d}}$ & 50 & $\mathrm{em}$ \\
$Q_{\mathrm{s}}$ & 36 & \\
$\alpha_{\mathrm{p}}$ & 0.55 & \\
$2 P$ & 40 & \\
$m$ & 3 & \\
\hline
\end{tabular}

\section{References}

1. Mallinson JC (1973) One-sided fluxes - a magnetic curiosity? IEEE Trans Magn 9(4):678-682

2. Halbach K (1979) Design of permanent multipole magnets with oriented rare earth cobalt material. Nucl Instrum Methods 169(1):110. https://doi.org/10.1016/0029-554X(80)90094-4

3. Chu W, Zhu Z, Shen Y (2013) Analytical optimisation of external rotor permanent magnet machines. IET Electr Syst Transp. https:// doi.org/10.1049/iet-est.2012.0045

4. Shen Y, Zhu Z-Q (2013) General analytical model for calculating electromagnetic performance of permanent magnet brushless machines having segmented Halbach array. J IET Electr Syst Transp. https://doi.org/10.1049/iet-est.2012.0055

5. Zhu ZQ, Xia ZP, Howe D (2002) Comparison of Halbach magnetized brushless machines based on discrete magnet segments or a single ring magnet. IEEE Trans Magn 38(5):2997-2999
6. Mellor PH, Wrobel R (2007) Optimization of a multipolar permanent magnet rotor comprising two arc segments per pole. IEEE Trans Ind Appl 43(4):942-951

7. Shen Y, Liu GY, Xia ZP, Zhu ZQ (2014) Determination of maximum electromagnetic torque in PM brushless machines having 2-segment Halbach array. IEEE Trans Ind Electron 61(2):718-729

8. Xia C, Guo L, Wang H (2014) Modeling and analyzing of magnetic field of segmented Halbach array permanent magnet machine considering gap between segments. IEEE Trans Magn 50(12):2-8

9. Kang H-B, Choi J, Cho H, Kim J (2014) Comparative study of torque analysis for synchronous permanent magnet coupling with parallel and Halbach magnetized magnets based on analytical field calculations. IEEE Trans Magn 50(11):1-3. https://doi.org/ 10.1109/TMAG.2014.2329010

10. Pristup AG et al (2014) A study of cogging torque in permanent magnet synchronous machines with fractional slot windings. Russ Electr Eng 85(12):36-40

11. Schmidt E, Susic M (2011) Finite element analysis of permanent magnet synchronous machines with fractional slot tooth coil windings. e \& i Elektrotech Informationstech 128(3):86-94. https://doi. org/10.1007/s00502-011-0812-z

12. Fateh $L$ et al (2016) Modeling and control of a permanent magnet synchronous generator dedicated to standalone wind energy conversion system. Front Energy 10(2):155-163. https://doi.org/10. 1007/s11708-016-0410-1

13. Del-Valle N et al (2011) Magnet guideways for superconducting maglevs: comparison between Halbach-type and conventional arrangements of permanent magnets. J Low Temp Phys 162:62-71. https://doi.org/10.1007/s10909-010-0225-0

14. Chin YK, Staton DA (2004) Transient thermal analysis using both lumped-circuit approach and finite element method of a permanent magnet traction motor. In: IEEE 7th AFRICON conference in Africa, vol 2, pp 1027-1035. https://doi.org/10.1109/AFRICON. 2004.1406847

15. Huang Y, Zhu J, Guo Y (2009) Thermal analysis of high-speed SMC motor based on thermal network and 3-D FEA with rotational core loss included. IEEE Trans Magn 45(10):4680-4682

16. Hendershot JR, Miller TJE (2010) Design of brushless permanentmagnet machines. Oxford University Press, Oxford, pp 157-193. ISBN 1-88155-03-1

17. Lipo TA (2011) Introduction to AC machine design. In: Library of congress cataloging in publication data. University of WisconsinMadison, Madison, pp 120-127

18. Krings A, Soulard J (2010) Overview and comparison of iron loss models for electrical machines. J Electr Eng 10(3):162-169

19. Ionel DM, Popescu M, Dellinger SJ, Miller TJE, Heideman RJ, McGilp MI (2006) On the variation with flux and frequency of the core loss coefficients in electrical machines. IEEE Trans Ind Appl 42(3):658-667

20. Ionel DM, Popescu M, McGilp MI, Miller TJE, Dellinger SJ, Heideman RJ (2007) Computation of core losses in electrical machines using improved models for laminated steel. IEEE Trans Ind Appl 43(6):1554-1564

21. Huang Y, Dong J, Zhu JG, Guo Y (2012) Core loss modeling for permanent-magnet motor based on flux variation locus and finite element method. IEEE Trans Magn 48(2):1023-1026

22. Meeker DC (2016) Finite element method magnetics, Version 4.2 (23 June 2016 Build). http://www.femm.info 